cloud, which are drawn out by the velocity of expansion without being mixed appreciably. There are two possible interpretations for the fact that the filaments are long but remain unmixed. One possibility is that there is a magnetic field, which prevents motions perpendicular to its own direction; the filaments would thus be similar in origin to coronal polar rays. The other possibility is that the mechanism which generates turbulence in most diffuse nebulae cannot operate in these objects, perhaps because they are bounded on only one side by denser material and are expanding into regions of very low density in the other directions.

Mount Wilson and Palomar Observatories, Pasadena, Calif.

\section{Philip, Kenelm W. Dispersion of short-wave radio pulses in interplanetary space.}

The existing, albeit meager, data for free electron density in interplanetary space suggest that a dispersion phenomenon will arise which may be detectable under some conditions. This dispersion, affecting group velocity as a function of frequency, could cause appreciable differences in the arrival time of short pulses, observed simultaneously at adjacent frequencies, which have traversed distances of the order of astronomical units in the solar system.

A calculation based on the best available data for the distribution of free electrons in the plane of the ecliptic shows that at I 5 megacycles, with a frequency separation of 500 kilocycles, this difference in arrival time would amount to $\frac{3}{4}$ millisecond over the path from earth to Jupiter at opposition. At conjunction, under the same conditions, the difference would be 20 milliseconds. These time delays should be measurable.

Since it seems probable that the reported millisecond pulses from Jupiter, and perhaps Venus, can be identified at frequencies separated by 500 kilocycles, this approach offers a possibility not only of verifying their extraterrestrial origin (a matter not yet conclusively settled), but also of direct measurement of electron densities out to the orbit of Jupiter.

Yale University Observatory, New Haven, Conn.

\section{Pillans, Helen and Struve, Otto. The spectrum of $\varepsilon$ Aurigae during eclipse.}

Striking differences in the spectrum of $\epsilon$ Aurigae occurred at ingress and at egress of the last eclipse. During the partial phase of ingress in September, October, and November, 1955 many strong lines with low excitation potential had sharp secondary components showing displacements toward the red of about $26 \mathrm{~km} / \mathrm{sec}$. The lines appeared strongly asymmetric or very broad but did not show a distinct doubling.

At the beginning of totality in December, I955 all the lines became symmetrical and sharp. The secondary component was nearly centrally placed on the original spectrum lines of the F star.

At the very end of totality, October and November, 1956, the strong lines of TiII, SrII, $S c I$, $C a$ I, and $F e$ I were distinctly double with velocities +ro $\mathrm{km} / \mathrm{sec}$ mostly from the $F$ star, and $-23 \mathrm{~km} / \mathrm{sec}$ mostly from the shell of the I-star. The strong $F e$ II lines were triple with an additional violet component at $-53 \mathrm{~km} / \mathrm{sec}$, also from the shell.

During the partial phase of egress in January, I957 the most remarkable change of all occurred. All of the strong lines, including $F e$ II, were double with velocities +8 and $-45 \mathrm{~km} / \mathrm{sec}$ but in addition a whole new spectrum of very sharp lines from levels of low excitation potential appeared. This new spectrum was rich in $F e$, $M n \mathrm{I}, C o \mathrm{I}, T i \mathrm{I}, C r \mathrm{I}, Y_{\mathrm{II}}, V_{\mathrm{II}}, S c \mathrm{II}, L a \mathrm{II}, N d \mathrm{II}$, TiII, and FeII. The highest energy level from which any of these lines originated was 2.7 volts. The lines originating from the ground levels of $F e I, C r I$, etc. were especially enhanced. The excitation temperature derived from the intensities of these sharp lines would be much lower than that of the F star or that derived from the shell components observed at other phases in the eclipse. According to unpublished measurements by Dr. M. Hack the turbulent velocity derived from the sharp lines is approximately $6 \mathrm{~km} / \mathrm{sec}$ or one half of that of the F-star lines. The velocity of the sharp lines was nearly the same as that of the violet components of the strong double lines.

It is probable that during totality the line of sight passed through several clouds located within the larger volume of space previously designated as the I-star and having different radial velocities. In January, I957, the line of sight passed through a single cloud whose ionization and excitation differed markedly from that of the clouds observed during totality.

Leuschner Observatory, University of California, Berkeley, Calif. 